\title{
Asymmetric Alkylation and Aldol Reactions of D-Mannitol-Derived Chiral Oxazolidin-2-one Derivatives
}

\author{
Yun Hee Maeng and Jong-Gab Jun* \\ Department of Chemistr. Hallym University; Chunchon 200-702, Korea \\ Recened September 19, 2003
}

Key Words : Oxazolidin-2-one, Chiral auxiliary. Alkylation, Aldol reaction, 1,2:5,6-Di-O-cyclohexylideneD-mannitol

In the preceeding article, we have introduced a new chiral oxazolidin-2-one auxiliary ( 1 ) derived from a cheap Dmannitol, and demonstrated the chiral selectivity in alkylation, aldol reaction and $\beta$-lactam synthesis.' The present work began with a search for useful chiral directing groups with which to control the chiral selectivity. Because the rigidity of cyclic structures contributes significantly to control of chirality, ${ }^{2}$ the 1,2:5,6-di-O-cyclohexylidene-Dmannitol (2) was used for the synthesis of oxazolidin-2-one chiral auxiliary (3) comparing the selectivity with the auxiliary (1) in alkylation and aldol reactions.<smiles>CC1(C)OCC([C@@H]2NC(=O)OC2[C@H]2COC(C)(C)O2)O1</smiles><smiles>OC(C(O)[C@H]1COC2(CCCCC2)O1)[C@H]1COC2(CCCCC2)O1</smiles>

1<smiles>O=C1N[C@H](C2COC3(CCCCC3)O2)[C@H](C2COC3(CCCCC3)O2)O1</smiles>

3
The 1,2:5,6-di-O-cyclohexylidene-D-mannitol (2), which was prepared from D-mannitol with cyclohexanone, boron trifluoride etherate and triethyl orthoformate in DMSO ${ }^{3}$ was converted into the cyclic sulfate $\mathbf{4}$ via cyclic sulfite methodology. This cyclic sulfate is similar to epoxide in that they undergo nucleophilic displacement $\left(S_{*}\right)$ readily, and produced 3-amino-3-deoxy-1,2:5,6-di-()-cyclohexyl-
idene-D-altritol (5) via azide displacement, hydrolysis followed by reduction (Scheme 1). The altritol 5 was converted into the chiral auxiliary 3 in $95 \%$ yield by using diethyl carbonate with sodium methoxide. ${ }^{6}$

The $N$-acylated derivatives 6 a-c were easily prepared in high yield by reaction of auxiliary 3 with acyl chlorides a-c using n-butyllithium in $\mathrm{TH} \Gamma$ at $-60^{\circ} \mathrm{C}$ (Table 1).

As we expected, I.DA mediated asymmetric alkylations of $\mathrm{N}$-acyl derivatives were obtained with high diastereomeric excess through 7.-enolate and re-face selectivity (Table 2). ${ }^{7}$

In most cases (entries al-cl except c2), the cyclohexylidene auxiliary 6 gave higher diastereomeric excess than the isopropylidene auxiliary derived from 1 (the \%de in parenthesis indicates the \%de from the isopropylidene auxiliary). The diasteromeric ratio was easily identified by the integration of benzyl (entries a1, b1, b2, c2) and allyl

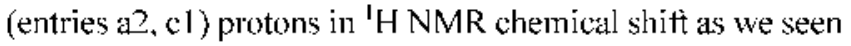
in previous results. Cyclohexylidene substituent in auxiliary

Table 1. N-Acylated derivatives 6a-c from the chiral auxiliary 3

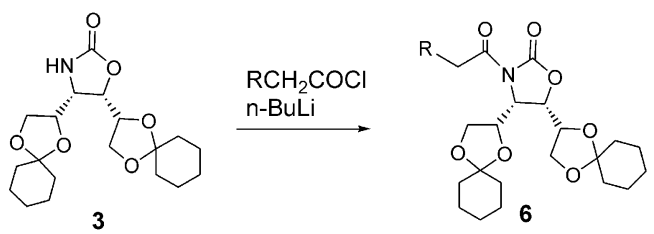

\begin{tabular}{ccccc}
\hline Entry & $R$ & Reaction time & Yield \% & $\begin{array}{c}{[\alpha]_{i}^{? 5}} \\
(\mathrm{c} . \mathrm{CHCl})\end{array}$ \\
\hline a & $\mathrm{CH}_{3}$ & $30 \mathrm{~min}$ & 92.2 & $+35.3(0.6)$ \\
$\mathrm{b}$ & $\mathrm{PhCH}_{2}$ & $30 \mathrm{~min}$ & 95.6 & $+33.3(1.2)$ \\
$\mathrm{c}$ & allyl & $30 \mathrm{~min}$ & 85.0 & $+32.9(1.1)$ \\
\hline
\end{tabular}

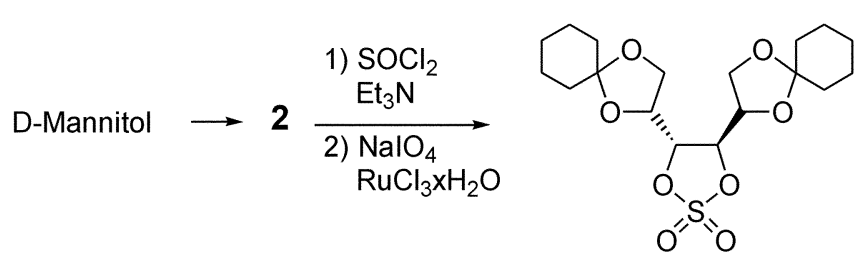

4

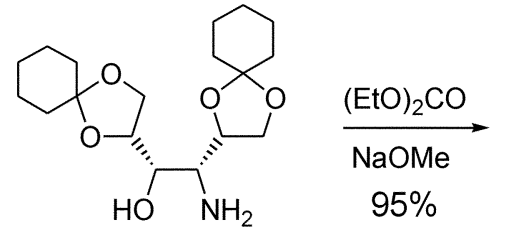

5

Scheme 1

\footnotetext{
"Corresponding author. Tel: +82-33-248-2075; F-mail: jgjun(ìnallym,ac.kr
} 
Table 2. Asymmetric alkylation of 's-acyl derivatives 6

\begin{tabular}{|c|c|c|c|c|c|c|}
\hline Entry: & $\mathrm{R}$ & $R^{\prime} X$ & $\operatorname{Rxn}(h)$ & $\%$ yicld ${ }^{\prime \prime}$ & $\% d c^{h}$ & {$[\alpha]_{D}$} \\
\hline al & $\mathrm{CII}_{3}$ & PhCII & 5 & 91.7 & $>99(94.0)$ & $\cdot 20.6(\mathrm{c} \quad 1.1,(\mathrm{CICl})$ \\
\hline 22 & $\mathrm{CII}_{2}$ & allyl hromide & 9 & 36.4 & $>99(91.6)$ & $\cdot 31.2\left(\mathrm{c} 1.7, \mathrm{ClOL}_{3}\right)$ \\
\hline hl & $\mathrm{PhCl}_{2}$ & Mel & 9 & 45.6 & $96.9(92.6)$ & $\cdot 53.8\left(\mathrm{c} \quad 1.9,\left(\mathrm{CH} / \mathrm{Cl}_{3}\right)\right.$ \\
\hline b2 & $\mathrm{PhCH}_{2}$ & allyl bromide & 6 & 55.6 & $96.8(91.6)$ & $-75.4\left(\mathrm{c}=1.1 . \mathrm{CHCl}_{3}\right)$ \\
\hline $\mathrm{cl}$ & allyl & $\mathrm{MeI}$ & 20 & 46.3 & $97.1(92.9)$ & $-52.2\left(\mathrm{c}=0.9 . \mathrm{CHCl}_{3}\right)$ \\
\hline$c 2$ & allyl & $\mathrm{PhCH}_{2} \mathrm{Br}$ & 20 & 52.1 & $89.4(96.7)$ & $-29.3\left(\mathrm{c}=1.1 . \mathrm{CHCl}_{\mathrm{j}}\right)$ \\
\hline
\end{tabular}

"Isolated yicld. "The \%de in parenthesis indicates the yield from the isopropylidene auxiliary' 1 .

shows bulkier and more ligid character in space than isopropylidene derivative, and gives better selectivity in alkylation.

We also applied this cyclohexylidene auxiliary $\mathbf{6}$ to the aldol reaction with benzaldehyde. "Evans" syn product $\mathbf{8}$ was obtained by using I equiv of ' $\mathrm{JiCl}_{4}$ via non-chelated $\angle$ enolate, however, "non-Evans" $s y n$ aldol product 9 was produced by using 2 equiv of $\mathrm{IiCl}_{4}$ via chelated $\angle$-enolate (Scheme 2). ${ }^{8}$ Selectivity employing $\mathrm{l}$ equiv of $\mathrm{CiCl}_{4}$ was >99: I Evans syn 8: non-Evans syn 9. The absolute configuration of $\mathbf{8}$ and the selectivity of syn:anti ratio were determined after hydrolytic cleavage of $\mathbf{8}$ to $\mathbf{1 0}$ by using $\mathrm{LiOOH} .{ }^{\circ}$ The hydrolysis gave $79.4 \%$ yield of $(2 S .3 .5)$-acid $10\left[[\alpha]_{[j}^{25}=-24.4\left(\mathrm{c}=0.9, \mathrm{CH}_{2} \mathrm{Cl}_{2}\right)\right.$, lit. $[\alpha]_{[2}^{22}=-26.4(\mathrm{c}=$ 1.04. $\left.\left.\mathrm{CH}_{2} \mathrm{Cl}_{2}\right)\right]^{10}$ with quantitative recovery $(\% 99 \%)$ of auxiliary 3. The ${ }^{\mathrm{H}} \mathrm{H}$ NMR of the product $\mathbf{1 0}$ indicated the selectivity $>96: 4$ for sy:nanti ratio similar to previous results.'

In the same way, we found that the selectivity for nonEvans sin 9 : Evans syn 8 employing 2 equiv of $\left[\mathrm{iCl} l_{+}\right.$was $>99: 1$ and for syn:anti of 11 after hydrolysis was $>82: 18$. No products from endocyclic cleavage in hydrolysis reaction were observed in both cases. "

In conclusion, the cyclohexylidene chiral auxiliary $\mathbf{3}$ derived from D-Inannitol shows better selectivity in asymmetric alkylations and comparable selectivity in aldol reactions compare with the isopropylidene derivative $\mathbf{l}$.

\section{Experimental Section}

All chemicals used were purchased from commercial sources and used as received unless otherwise stated. NMR spectra were recorded at Varian Gemini- $400 \mathrm{MHz}$ F [-NMR for ${ }^{1} \mathrm{H}$ and $100 \mathrm{MHz}$ for ${ }^{1.3} \mathrm{C}$, with the chemical shifts $(\delta)$ reported in parts per million (ppm) relative to l'MS and the coupling constants $(J)$ quoted in $\mathrm{Hz} . \mathrm{CDCl}_{\text {; was }}$ wsed as a solvent and an internal standard. Infrared spectra were recorded on a Shimadzu JR-435 spectrometer. GC-MS analyses were performed using a H3-5890/JMS-AM 150. JEOL. Flash chromatography was carried out using silica gel Merck 60 (230-400 mesh). Thin-layer chromatography (ILC) was performed on DC-Plastikfolien 60. Fis4 (Merck, layer thickness $0.2 \mathrm{~mm}$ ) plastic-backed silica gel plates with visualization by UV light (254 nın) or by treatment with $p$ anisaldehyde. Melting points were measured on a MEL[EMP' II apparatus and were uncorrected.

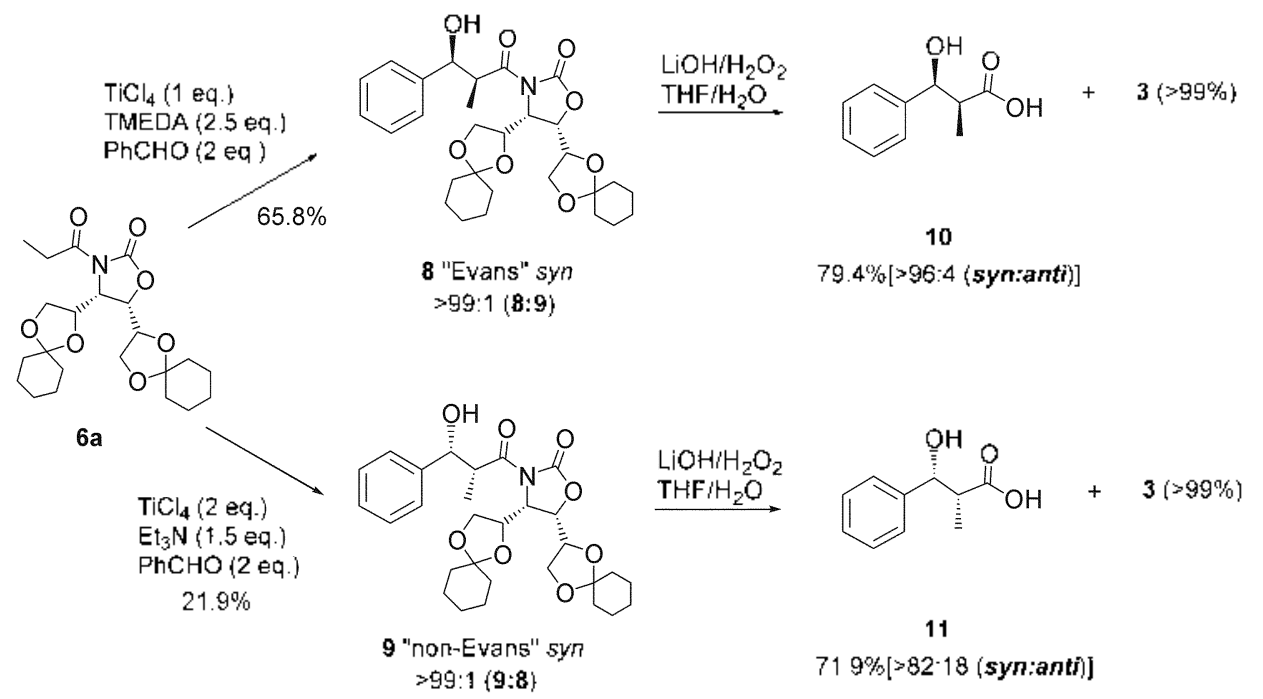

Scheme 2 
(4S,5R)-4,5-Bis-(2,2-dicyclohexyl-1,3-dioxolan-4-yl)oxazolidin-2-one (3). To a solution of 3-amino-3-deosy1,2:5.6-di-O-isopropylidene-D-altritol $\quad(5) \quad(0.63$ g. $\quad 1.85$ inmol) in diethyl carbonate $(3.15 \mathrm{~mL})$ under nitrogen atmosphere was added sodium methoxide $(0.11 \mathrm{~mL}$ of $25 \%$ solution in $\mathrm{MeOH}$. 0.46 mumol) and heated for $3 \mathrm{~h}$ at $70-80$ ${ }^{\circ} \mathrm{C}$. Diethyl carbonate was removed by evaporation and the residual solid was washed with hexane, recrystallized by $\mathrm{MeOH}$ to give the white solid $3(0.64$ g. $95 \%) . R_{\mathrm{f}} 0.47$ $\left(\mathrm{MeOH}: \mathrm{CHCl}_{3}=1: 9\right) ; m p 170-172{ }^{\circ} \mathrm{C}:[\alpha]_{\mathrm{D}}^{20}-35.8(c 1.0$. $\mathrm{CHCl}_{j}$ ): $V_{\text {max }}($ film $) / \mathrm{cm}^{-1} 3288,2933,2850,1757.1738$. 1094: ${ }^{1} \mathrm{H}$ NMR $\left(400 \mathrm{MHz}, \mathrm{CDCl}_{3}\right) \delta 1.40-1.66(20 \mathrm{H} . \mathrm{m})$. $3.79(\mathrm{lH}$, dd. $J 9.3 .4 .6 \mathrm{~Hz}$ ). $3.83-3.88(\mathrm{lH} . \mathrm{m}) .3 .95 \cdot 3.99$ $(1 \mathrm{H}, \mathrm{m}) .4 .13-4.19(2 \mathrm{H}, \mathrm{m}) .4 .33-4.39(2 \mathrm{H} . \mathrm{m}), 4.40-4.46$ $(1 \mathrm{H}, \mathrm{m}) .5 .43(\mathrm{H}$, br s. $\mathrm{NH}) ;{ }^{13} \mathrm{C}$ NMR $\left(100 \mathrm{MHz}, \mathrm{CDCl}_{3}\right)$ 158.1. 111.2. 110.8. 78.1. 73.9, 71.9, 67.7. 67.1, 58.3, 36.9 . $36.7,34.9 .34 .6,25.4 .25 .3,24.4(\times 2), 24.2,24.1$.

Typical Procedure for the Preparation of $\mathrm{N}$-Acyloxazolidin-2-ones, 6a-c. To a solution of oxazolidinone 3 (1.00 g. $2.72 \mathrm{mmol})$ in THF ( $100 \mathrm{~mL})$ under nitrogen atmosphere was added $n-\mathrm{BuLi}(2.55 \mathrm{~mL}$ of $1.6 \mathrm{M}$ solution in Hexane. $4.08 \mathrm{mmol}$ ) at $-60^{\circ} \mathrm{C}$ and stirred for $30 \mathrm{~min}$. Propionyl chloride $(0.47 \mathrm{~mL} .5 .44 \mathrm{mmol})$ was added to this reaction mixture at $-40^{\circ} \mathrm{C}$ and stirred for $30 \mathrm{~min}$. The reaction was quenched by the addition of water at $0{ }^{\circ} \mathrm{C}$. The organic product was extracted with ethyl acetate. washed with brine. dried, concentrated and chromatographed (EtOAc: $\mathrm{Hex}=$ $1: 4)$ to give the liquid $6 \mathrm{a}(1.06 \mathrm{~g}, 92.2 \%)$.

$(+S, 5 R)$-3-(1-Oxopropyl)- $4,5-$ bis-(2,2-dicyclohexyl-1,3dioxolan-4-yl)-oxazolidin-2-one (6a). $R_{\mathrm{f}} 0.42$ (EtOAC : Hex $=1: 4):[\alpha]_{\mathrm{D}}^{10}+35.3\left(c \quad 0.6 . \mathrm{CHCl}_{3}\right):{ }^{1} \mathrm{H}$ NMR $(400 \mathrm{MHz}$. $\left.\mathrm{CDCl}_{3}\right) \delta 1.18(3 \mathrm{H}$. t. $J 7.3 \mathrm{~Hz}), 1.29-1.58(20 \mathrm{H.} \mathrm{m}), 3.92 \cdot$ $4.06(3 \mathrm{H} . \mathrm{m}) .4 .18(\mathrm{lH}, \mathrm{dd} . J 9.2 .5 .8 \mathrm{~Hz}) .4 .3 \mathrm{l}(1 \mathrm{H}, \mathrm{dd} . J$ $9.8 .7 .0 \mathrm{~Hz}), 4.58-4.68(2 \mathrm{H.} \mathrm{m}), 4.73(\mathrm{lH} . \mathrm{d} . J 6.7 \mathrm{~Hz})$.

$(+S, 5 R)$-3-(3-Phenyl-1-0xopropyl)-4,5-bis-(2,2-dicyclohexyl-1,3-dioxolan-4-yl)-oxazolidin-2-one (6b). 95.6\%; $R_{\mathrm{f}}$ 0.45 (EtOAc : Hex $=1 ; 4):[\alpha]_{D}^{20}+33.3\left(c \quad 1.2, \mathrm{CHCl}_{3}\right) ;{ }^{1} \mathrm{H}$ NMR $\left(400 \mathrm{MHz} . \mathrm{CDCl}_{3}\right) \delta 1.29-1.67(20 \mathrm{H} . \mathrm{m}) .2 .94-3.11(2 \mathrm{H}$. m) $3.20-3.29(2 \mathrm{H} . \mathrm{m}) .3 .87-4.05(3 \mathrm{H}, \mathrm{m}) .4 .10-4.29(2 \mathrm{H}$. m). $4.56-4.65(2 \mathrm{H} . \mathrm{m}) .4 .70(1 \mathrm{H} . \mathrm{d} . J 6.7 \mathrm{~Hz}) .7 .15-7.32(5 \mathrm{H} . \mathrm{m})$.

$(4 S, 5 R)$-3-(1-Oxo-4-pentenyl)-4,5-bis-(2,2-dicyclohexyl1,3-dioxolan-4-yl)-oxazolidin-2-one (6c). $85.0 \%: R_{\mathrm{f}} 0.52$ (EtOAc : $\left.\mathrm{Hex}=1: 4):[\alpha]_{\mathrm{D}}^{20}+32.9(c) 1.1 . \mathrm{CHCl}_{3}\right):{ }^{1} \mathrm{H}$ NMR $\left(400 \mathrm{MHz} . \mathrm{CDCl}_{3}\right) \delta 1.39-1.72(20 \mathrm{H} . \mathrm{m}), 2.37-2.49(2 \mathrm{H} . \mathrm{m})$. 2.96-3.07 (2H. m). 3.91-4.13 (3H. m) $4.18(1 \mathrm{H} . \mathrm{dd} J 7.5$. $3.4 \mathrm{~Hz}$ ). $4.30(1 \mathrm{H}$. dd $J 9.8 .8 .4 \mathrm{~Hz}), 4.58-4.69(2 \mathrm{H} . \mathrm{m}) .4 .72$ $(1 \mathrm{H}$ d $J 6.7 \mathrm{~Hz}) .4 .99-5.14(2 \mathrm{H} . \mathrm{m}) .5 .79-5.88(1 \mathrm{H} . \mathrm{m})$

Typical Procedure for the Preparation of Alkylated Products, 7al-7e2. To a solution of diisopropyl amine $(0.05$ $\mathrm{mL}, 0.35 \mathrm{mmol})$ in THF $(3 \mathrm{~mL})$ at $-20^{\circ} \mathrm{C}$ under nitrogen atmosphere was added $n-\mathrm{BuLi}(0.22 \mathrm{~mL}$ of $1.6 \mathrm{M}$ solution in Hexane. $0.35 \mathrm{~mm}$ mol) and stirred for $30 \mathrm{~min} . \mathrm{N}$-Propionyl oxazolidinone $6 \mathrm{a}(0.10 \mathrm{~g} .0 .24 \mathrm{mmol})$ in THF $(2 \mathrm{~mL})$ was added to this reaction mixture at $-60^{\circ} \mathrm{C}$ and stirred for 30 min. Benzyl bronide $(0.11 \mathrm{~mL} .0 .94 \mathrm{mmol})$ was added to this reaction mixture at $-40^{\circ} \mathrm{C}$ and stirred for $2 \mathrm{~h}$. The reaction was quenched by the addition of water at $0^{\circ} \mathrm{C}$. The organic product was extracted with ethyl acetate. washed with brine, dried, concentrated, and chromatographed (EtOAc Hex $=1: 4)$ to give the liquid 7 a1 $(0.11 \mathrm{~g} .91 .7 \%)$

$\left(4 S, 5 R, 2^{\prime} R\right)$-3-(2-Methyl-3-pheny-l-oxopropyl)-4,5-bis(2,2-dicyclohexyl-1,3-dioxolan-t-yl)-oxazolidin-2-one (7a1). $R_{\mathrm{f}} 0.45$ (EtOAc: $\mathrm{Hex}=1: 4$ ); $[\alpha]_{\mathrm{D}}^{20}+20.6(c$ l.1, $\left.\mathrm{CHCl}_{3}\right) ;{ }^{1} \mathrm{H}$ NMR $\left(400 \mathrm{MHz} . \mathrm{CDCl}_{3}\right) \delta 1.10(3 \mathrm{H}, \mathrm{d}, J 6.8$ $\mathrm{Hz}, \alpha$-methyl), $1.17 \cdot 1.55(20 \mathrm{H}, \mathrm{m}), 2.50(1 \mathrm{H}, \mathrm{dd}, J 13.3,8.4$ Hz. benzyl proton), 3.18 (lH. dd. $J 13.2,6.2 \mathrm{~Hz}$. benzyl proton), $3.47(\mathrm{lH}$, dd. $J 8.9,7.3 \mathrm{~Hz}), 3.87(\mathrm{lH}$. dd. $J 9.0 .6 .3$ $\mathrm{Hz}), 3.91-3.98(2 \mathrm{H}, \mathrm{m}), 4.11(\mathrm{lH} . \mathrm{dd}, J 9.1,5.8 \mathrm{~Hz}), 4.25$ (IH. dd. $J 9.7 .6 .9 \mathrm{~Hz}), 4.47$ (lH, t. $J 6.7 \mathrm{~Hz}) .4 .57(\mathrm{IH} . \mathrm{m}$, $\alpha$-proton), $4.66(\mathrm{lH}$. d. $J 7.0 \mathrm{~Hz}), 7.13(\mathrm{lH} . \mathrm{m}), 7.19-7.21$ $(4 \mathrm{H} . \mathrm{m}) ;{ }^{13} \mathrm{C} \mathrm{NMR}\left(100 \mathrm{MHz} . \mathrm{CDCl}_{3}\right) \delta 175.5(\mathrm{C}=0) .151 .9$ $(\mathrm{C}=0) .138 .2,128.3(\mathrm{x} 2), 127.3(\mathrm{x} 2), 125.3,109.8,108.9$, 76.1. 72.7.71.3. 66.0. 64.5. 54.4. 28.6 .38 .5 .35 .9 .34 .3 .34 .0 . $33.5,24.0 .23 .9,23.0 .22 .9 .22 .8,22.7 .15 .4$

(4S,5 $\left.R, 2^{\prime} R\right)$-3-(2-Methyl-1-ox0-t-pentenyl)-4,5-bis-(2,2dicyclohexyl-1,3-dioxolan-t-yl)-oxazolidin-2-one (7a2). $36.4 \% ; R_{\mathrm{f}} 0.48$ (EtOAc: Hex $\left.=1: 4\right):[\alpha]_{\mathrm{D}}^{20}+31.2(c 1.7$, $\left.\mathrm{CHCl}_{3}\right) ;{ }^{1} \mathrm{H}$ NMR $\left(400 \mathrm{MHz} . \mathrm{CDCl}_{3}\right) \delta 1.13(3 \mathrm{H}, \mathrm{d}, J 6.8$ $\mathrm{Hz}, \alpha$-metlyl). 1.37-1.63 $(20 \mathrm{H}, \mathrm{m}) .2 .19(\mathrm{lH}, \mathrm{m}$. allyl proton), $2.59(\mathrm{lH}, \mathrm{m}$, allyl proton), $3.77(\mathrm{lH}$, quintet. $J 6.7$ $\mathrm{Hz}), 3.84(\mathrm{lH}, \mathrm{dd}, J 8.9 .6 .7 \mathrm{~Hz}) .3 .99-4.05(2 \mathrm{H} . \mathrm{m}), 4.18$ (IH. dd. $J 9.1,5.9 \mathrm{~Hz}) .4 .31$ (lH. dd. $J 9.7,6.9 \mathrm{~Hz}), 4.60$ (lH. t. $J 6.6 \mathrm{~Hz}) .4 .65(\mathrm{lH}, \mathrm{m}) .4 .73(\mathrm{lH}, \mathrm{d} . J 6.9 \mathrm{~Hz}) .5 .06$ $(\mathrm{lH} . \mathrm{m},=\mathrm{CH}$ trans). $5.12(\mathrm{lH} . \mathrm{s},=\mathrm{CH}$ cis $), 5.80(1 \mathrm{H}, \mathrm{m}$, $=\mathrm{CH}$ intemal $):{ }^{13} \mathrm{C}$ NMR $\left(100 \mathrm{MHz}, \mathrm{CDCl}_{3}\right) \delta 176.7$ $(C=O), 152.9(\mathrm{C}=0) .135 .3 .117 .2110 .8,110.1,77.1 .73 .8$. 72.3.67.1.65.6. 55.8.37.8.37.1.37.0.35.5.34.8.34.6.25.1. $25.0,24.1 .23 .9,23.8(\mathrm{x} 2), 16.4$

$(4 S, 5 R, 2 ' S)$-3-(2-Methyl-3-pheny-l-oxopropyl)-4,5-bis(2,2-dicyclohexyl-1,3-dioxolan-4-yl)-oxazolidlin-2-one (7b1). $45.6 \% ; R_{\mathrm{f}} 0.52$ (EtOAc: Hex $\left.=1: 4\right):[\alpha]_{\mathrm{D}}^{20}+53.8$ (c 1.9 , $\left.\mathrm{CHCl}_{3}\right) ;{ }^{1} \mathrm{H}$ NMR $\left(400 \mathrm{MHz} . \mathrm{CDCl}_{3}\right) \delta 1.27(3 \mathrm{H}, \mathrm{d}, J 6.8$ Hz. $\alpha$-methyl), $1.36-1.63(20 \mathrm{H} . \mathrm{m}), 2.72(\mathrm{lH}, \mathrm{dd}, J 13.3,7.0$ $\mathrm{Hz}$. benzyl proton). 2.93 (IH. dd. $J 13.4,8.2 \mathrm{~Hz}$, benzyl proton), $3.87(2 \mathrm{H} . \mathrm{dd} . J 9.3 .6 .5 \mathrm{~Hz}) .3 .94 \mathrm{dd} . J 9.0 .3 .4 \mathrm{~Hz}) .4 .00$ (1H. dd. $J 9.0 .6 .4 \mathrm{~Hz}), 4.08-4.14(2 \mathrm{H}, \mathrm{m}) .4 .52-4.57(3 \mathrm{H}$. m), 7.17-7.28 (5H. m): ${ }^{13} \mathrm{C}$ NMR (100 MHz. $\left.\mathrm{CDCl}_{2}\right) \delta 176.8$ $(C=O), 152.9(\mathrm{C}=0) .139 .1$. $129.1(\mathrm{x} 2), 128.4(\mathrm{x} 2), 126.4$. $110.8 .110 .1,77.0 .73 .8 .72 .2 .67 .0 .65 .7,55.8,40.1,38.9$. $36.9,35.4,34.7,34.5,25.0 .24 .9,24.0 .23 .9,23.8(\mathrm{x} 2), 17.6$.

(4S,5R,2'S)-3-(2-Benzyl-l-ox0-4-pentenyl)-4,5-bis-(2,2dicyclohexyl-1,3-dioxolan-4-yl)-oxazolidin-2-one (7b2). 55.6\%: $R_{\mathrm{f}} 0.53($ EtOAc : Hex $=1: 4):[\alpha]_{\mathrm{D}}^{20}+75.4$ (c 1.1. $\left.\mathrm{CHCl}_{3}\right):{ }^{1} \mathrm{H}$ NMR $\left(400 \mathrm{MHz}, \mathrm{CDCl}_{3}\right) \delta 1.42-1.60(20 \mathrm{H}, \mathrm{m})$. 2.35 ( $1 \mathrm{H}$. m. allyl proton). 2.60 ( $1 \mathrm{H}$. m. allyl proton). 2.76 (1H. dd. $J 13.0 .100 \mathrm{~Hz}$. benzyl proton). 2.87 (1H. dd. $J$ 13.0. $6.1 \mathrm{~Hz}$. benzyl proton), $3.47(1 \mathrm{H}$. dd. $J 9.8,6.8 \mathrm{~Hz}$ ). $3.76(1 \mathrm{H}$ br t. $J 7.6 \mathrm{~Hz}), 3.85(1 \mathrm{H}$. dd $J 9.1 .3 .4 \mathrm{~Hz}) .3 .97$ $(1 \mathrm{H} . \mathrm{dd}, J 8.9 .6 .5 \mathrm{~Hz}) .4 .08(1 \mathrm{H} . \mathrm{dd} J 9.1,5.9 \mathrm{~Hz}) .4 .31$ $(2 \mathrm{H} . \mathrm{m}) .4 .46(2 \mathrm{H} . \mathrm{m}) .5 .04(1 \mathrm{H}$. br d $J 10.3 \mathrm{~Hz} .=\mathrm{CH}$ trans $)$. $5.14(1 \mathrm{H}$, d. $J 16.4 \mathrm{~Hz} .=\mathrm{CH} c i s) .5 .84(1 \mathrm{H}, \mathrm{m} .=\mathrm{CH}$ intemal). $7.15(2 \mathrm{H}, \mathrm{m}), 7.19-7.27(3 \mathrm{H}, \mathrm{m})$.

(4S,5R,2'S)-3-(2-Methyl-1-0xo-4-pentenyl)-4,5-bis-(2,2dicyclohexyl-1,3-dioxolan-4-yl)-oxazolidin-2-one (7c1). 
$46.3 \% ; R_{\mathrm{f}} 0.59$ (EtOAc: Hex $\left.=1: 4\right) ;[\alpha]_{\mathrm{D}}^{20}+52.2$ (c 0.9 . $\left.\mathrm{CHCl}_{3}\right):{ }^{\mathrm{H}} \mathrm{H} \mathrm{NMR}\left(400 \mathrm{MHz}, \mathrm{CDCl}_{3}\right) \delta 1.25(3 \mathrm{H} . \mathrm{d} . J 6.6$ $\mathrm{Hz}, \alpha$-methyl). 1.37-1.59 (20H. m), 2.17 (lH. m. allyl proton $), 2.40(1 \mathrm{H}, \mathrm{m}$. allyl proton $), 3.79(1 \mathrm{H}, \mathrm{m}) .3 .87(\mathrm{lH}$. dd. $J 9.0 .6 .5 \mathrm{~Hz}) .4 .03(2 \mathrm{H}, \mathrm{ml}) .4 .18(1 \mathrm{H}, \mathrm{dd}, J 9.2 .5 .9 \mathrm{~Hz})$. $4.28(1 \mathrm{H}, \mathrm{dd}, J 9.8 .6 .9 \mathrm{~Hz}) .4 .58-4.66(2 \mathrm{H}, \mathrm{m}), 4.73(\mathrm{lH} . \mathrm{d}$. $J 6.9 \mathrm{~Hz}), 5.01(\mathrm{lH} . \mathrm{ml} .=\mathrm{CH} t r a n s) .5 .07(\mathrm{lH} . \mathrm{s},=\mathrm{CH} c i s)$. $5.75(\mathrm{lH} . \mathrm{m},=\mathrm{CH}$ intemal $)$.

$\left(+S, 5 R, 2^{\prime} R\right)$-3-(2-Benzyl-1-oxo-4-pentenyl)-4,5-bis-(2,2dicyclohexyl-1,3-dioxolan-4-yl)-oxazolidin-2-one (7c2). $52.1 \% ; R_{\mathrm{f}} 0.56$ (EtOAc: Hex $\left.=1: 4\right) ;[\alpha]_{\mathrm{D}}^{20}+29.3$ (c l.1. $\left.\mathrm{CHCl}_{3}\right):{ }^{1} \mathrm{H}$ NMR (400 MHz. $\left.\mathrm{CDCl}_{3}\right) \delta 1.52-1.59$ (20H. m). 2.24 ( $\mathrm{H}$. m, allyl proton). 2.34 ( $1 \mathrm{H}$, m. allyl proton). 2.71 $(1 \mathrm{H}, \mathrm{dd}, J 13.5,7.2 \mathrm{~Hz}$. benzyl proton $), 3.16(1 \mathrm{H}, \mathrm{dd}, J 13.5$. $7.5 \mathrm{~Hz}$, benzyl proton), $3.43(\mathrm{lH}$. dd. $J 8.8 .7 .8 \mathrm{~Hz}$ ). $3.9 \mathrm{l}$ $(1 \mathrm{H}$, dd. $J 9.0,6.1 \mathrm{~Hz}), 4.02(1 \mathrm{H}$, dd. $J 9.1 .3 .5 \mathrm{~Hz}), 4.16$ $(1 \mathrm{H}, \mathrm{dd}, J 9.1 .5 .9 \mathrm{~Hz}), 4.20-4.25(2 \mathrm{H} . \mathrm{m}), 4.49(1 \mathrm{H}, \mathrm{br} \mathrm{t} . J$ $7.2 \mathrm{~Hz}) .4 .60(1 \mathrm{H}, \mathrm{m}), 4.67(1 \mathrm{H}, \mathrm{dd}, J 7.3 .0 .9 \mathrm{~Hz}), 4.99(\mathrm{lH}$. $\mathrm{s},=\mathrm{CH} c i s), 5.03(\mathrm{lH} . \mathrm{d}, J 5.9 \mathrm{~Hz},=\mathrm{CH} t /(m s) .5 .73(\mathrm{lH}, \mathrm{m}$. $=\mathrm{CH}$ intemal $), 7.18(1 \mathrm{H}, \mathrm{m}) \cdot 7.23-7.27(4 \mathrm{H} . \mathrm{m})$.

$(+S, 5 R, 2 ' S, 3 ' S)$-3-(3-Hydroxy-2-methyl-3-pheny-l-oxopropyl)-4,5-bis-(2,2-dicyclohexyl-1,3-dioxolan-t-yl)-oxazolidin-2-one (8). To a solution of $(4 S .5 R)-3$-(1-oxopropyl)4,5-bis-(2,2-dicyclohexyl-1,3-dioxolan-4-yl)-oxazolidin-2one (6a) $(0.15 \mathrm{~g} .0 .35 \mathrm{mmol})$ in $\mathrm{CH}_{2} \mathrm{Cl}_{2}(5 \mathrm{~mL})$ under nitrogen atmosphere was added $\mathrm{TiCl}_{4}(0.39 \mathrm{~mL}$ in $1.0 \mathrm{M}$ solution in $\mathrm{CH}_{2} \mathrm{Cl}_{2}, 0.39 \mathrm{mmol}$ ) at $-60^{\circ} \mathrm{C}$ and stirred for 5 min. TMEDA $(0.13 \mathrm{~mL} .0 .89 \mathrm{mmol})$ was added to this reaction mixture at $-60{ }^{\circ} \mathrm{C}$ and stirred for $30 \mathrm{~min}$. Benzaldehyde $(0.07 \mathrm{~mL}, 0.71 \mathrm{mmol})$ was added to this reaction mixture at $-60^{\circ} \mathrm{C}$ and stirred for $2 \mathrm{~h}$. The reaction was quenched by the addition of $50 \%$ aqueous $\mathrm{NH}_{4} \mathrm{Cl}$ at $0{ }^{\circ} \mathrm{C}$. The organic product was extracted with ethyl acetate. washed with brine. dried and concentrated to give the product 8 ( 53 mg. $65.8 \%) \cdot R_{\mathrm{f}} 0.3 \mathrm{l}$ (EtOAc: $\left.\mathrm{Hex}=1: 2\right)$. ${ }^{1} \mathrm{H} \mathrm{NMR}(400 \mathrm{MHz}$, $\left.\mathrm{CDCl}_{3}\right) \delta 1.27(3 \mathrm{H} . \mathrm{d}, J 6.9 \mathrm{~Hz}, \alpha$-methyl). 1.34-1.57 (20H. m) $, 3.33(\mathrm{lH} . \mathrm{d},, J 3.0 \mathrm{~Hz}), 3.83-3.93(3 \mathrm{H}, \mathrm{ml}), 4.01-4.14$ $(3 \mathrm{H} . \mathrm{m}) .4 .92(1 \mathrm{H} . \mathrm{dd} . J 5.0 .28 \mathrm{~Hz}) .7 .24-7.32(5 \mathrm{H} . \mathrm{m})$.

( $\left.4 S, 5 R, 2^{\prime} R, 3^{\prime} R\right)$-3-(3-Hydroxy-2-methyl-3-pheny-l-oxopropyl)-4,5-bis-(2,2-dicyclohexyl-1,3-dioxolan-4-yl)-oxazolidin-2-one (9). To a solution of (4S.5R)-3-(1-oxopropy 1)4.5-bis-(2.2-dimethyl-1.3-dioxolan-4-yl)-oxazolidin-2-one (6a) $(0.15 \mathrm{~g} .0 .35 \mathrm{mmol})$ in $\mathrm{CH}_{3} \mathrm{Cl}_{2}(7 \mathrm{~mL})$ under nitrogen atmosphere was added $\mathrm{TiCl}_{4}(0.71 \mathrm{~mL}$ in $1.0 \mathrm{M}$ solution in $\mathrm{CH}_{2} \mathrm{Cl}_{-} .0 .71 \mathrm{mmol}$ ) at $-60{ }^{\circ} \mathrm{C}$ and stirred for $5 \mathrm{~min}$. $\mathrm{Et}_{3} \mathrm{~N}$ $(0.07 \mathrm{~mL}, 0.53 \mathrm{mmol})$ was added to this reaction mixture at $-60^{\circ} \mathrm{C}$ and stirred for $30 \mathrm{~min}$. Benzaldehyde $(0.07 \mathrm{~mL} .0 .71$ mmol) was added to this reaction mixture at $-60^{\circ} \mathrm{C}$ and stirred for $2 \mathrm{~h}$. The reaction was quenched by the addition of water at $0^{\circ} \mathrm{C}$. The organic product was extracted with ethyl acetate. washed with brine. dried and concentrated to give the product 9 (4l mg. $21.9 \%$ ). $R_{\mathrm{f}} 0.43$ (EtOAc : Hex $=1: 2$ ): ${ }^{1} \mathrm{H}$ NMR $\left(400 \mathrm{MHz} . \mathrm{CDCl}_{3}\right) \delta 0.96(3 \mathrm{H}$. d. $J 6.9 \mathrm{~Hz} . \alpha-$ methyl). $1.37-1.60(20 \mathrm{H} . \mathrm{m}) .3 .51(1 \mathrm{H} . \mathrm{d} . J 4.7 \mathrm{~Hz}) .3 .95-$ $4.12(4 \mathrm{H}, \mathrm{m}) .4 .15-4.21(2 \mathrm{H}, \mathrm{m}) .4 .32-4.37(1 \mathrm{H}, \mathrm{m}), 4.67-$ $4.70(2 \mathrm{H}, \mathrm{m}) .4 .88(1 \mathrm{H} . \mathrm{dd}, J 7.1,1.1 \mathrm{~Hz}), 7.24-7.37(3 \mathrm{H}$. m). $7.46(2 \mathrm{H} . \mathrm{d} . J 6.9 \mathrm{~Hz})$.
Syn-(2S,3S)- and anti-(2R,3S)-3-Hydloxy-2-methyl-3phenylpropanoic acid (10). To a solution of $\left(4, S, 5 R, 22^{\prime} S, 3^{\prime} S\right)$ 3-(3-hydroxy-2-methyl-3-pheny-l-oxopropyl)-4.5-bis-(2.2dicyclohexyl-1.3-dioxolan-4-yl)-oxazolidin-2-one (8) (100 mg. $0.19 \mathrm{mmol})$ in THF $(2.9 \mathrm{~mL})$ and $\mathrm{H}_{2} \mathrm{O}(0.95 \mathrm{~mL})$ was added $30 \% \mathrm{H}_{2} \mathrm{O}_{2}(1.07 \mathrm{~g} .0 .94 \mathrm{mmol})$ and $\mathrm{LiOH} \cdot \mathrm{H}_{2} \mathrm{O}$ (I6 mg. $0.38 \mathrm{mmol}$ ) at $0^{\circ} \mathrm{C}$ and stirred for $30 \mathrm{~min}$. Solid sodium sulfite and saturated $\mathrm{NaHCO}_{3}$ solution were added to this reaction mixture until $\mathrm{pH} 10$. THF in the reaction mixture was evaporated. The mixture was diluted with water $(2.5$ $\mathrm{mL}$ ), extracted with $\mathrm{CH}_{2} \mathrm{Cl}_{2}$, washed with brine, dried and concentrated to give the auxiliary $3(70 \mathrm{mg}$. $100 \%$ ). The water layer was acidified with the addition of $3 \mathrm{~N} \mathrm{HCl}$ solution until $\mathrm{pH} 2$, and extracted with EtOAc, washed with brine. dried, concentrated and cluromatographed to give the acids $10\left(27 \mathrm{mg} .79 .4 \%\right.$ ). $R_{\mathrm{f}} 0.19$ (EtOAc $: \mathrm{Hex}=1: 2$ ): $\left.[\alpha]_{\mathrm{D}}^{25}-24.4\left(c 0.9 . \mathrm{CH}_{2} \mathrm{Cl}\right)_{2}\right)$ : [lit. ${ }^{16}[\alpha]_{\mathrm{D}}^{22}=-26.4(c) 1.04$. $\left.\mathrm{CH}_{2} \mathrm{Cl}_{2}\right)$ ]: ${ }^{1} \mathrm{H} \mathrm{NMR}\left(400 \mathrm{MHz}, \mathrm{CDCl}_{3}\right) \delta 1.14(3 \mathrm{H}$, d. $J 9.0$ $\mathrm{Hz}, \alpha$-methyl). $2.83(\mathrm{lH} . \mathrm{m} . \alpha$-H), $4.75(0.0 \mathrm{lH}$. d, $J 8.8 \mathrm{~Hz}$, anti $\mathrm{CHOH}) .5 .18(0.99 \mathrm{H}, \mathrm{d}, J 4.0 \mathrm{~Hz} .5 n \mathrm{CHOH}) .5 .42$ (2H. br s. $\mathrm{OH}$ and $\left.\mathrm{CO}_{2} \mathrm{H}\right) .7 .35\left(5 \mathrm{H}\right.$, s. aromatic). ${ }^{1} \mathrm{H}$ NMR integration afforded a ratio syn:anti $=96: 4$. The data were consistent with those reported in the literature. ${ }^{10112}$

Syn-(2R,3R)- and anti-(2S,3R)-3-Hydloxy-2-methyl-3phenylpropanoic acid (11). Prepared from 9 (4l mg. 0.08 minol) as same as above procedure and gave the acids 11 (10 $\mathrm{mg} .71 .9 \%)$ and the auxiliary $3\left(28 \mathrm{mg} .100 \%\right.$ ). $R_{\mathrm{f}} 0.19$ (EtOAc : Hex = $1: 2):[\alpha]_{\mathrm{D}}^{35}+26.5\left(c 0.35, \mathrm{CH}_{3} \mathrm{Cl}_{2}\right) ;\left[\right.$ lit. ${ }^{101}$ $\left.[\alpha]_{\mathrm{D}}^{12}=-26.4\left(c 1.04 . \mathrm{CH}_{2} \mathrm{Cl}_{2}\right)\right]$ for the entantiomer 10. ${ }^{1} \mathrm{H}$ NMR $\left(400 \mathrm{MHz}, \mathrm{CDCl}_{3}\right) \delta 4.75(0.04 \mathrm{H}, \mathrm{d}, J 8.8 \mathrm{~Hz}$, anti $\mathrm{CHOH}), 5.18(0.96 \mathrm{H} . \mathrm{d}, J 4.0 \mathrm{~Hz} . \mathrm{sm} \mathrm{CHOH}) .{ }^{1} \mathrm{H}$ NMR integration afforded a ratio stn:anti $=82: 18$.

Acknowledgment. This work was supported by the Research Grant from Hallym University. Korea.

\section{References}

1. (a) Kim. S.-M.: Jin. H.: Jun, J.-G. Bull. Konan Chem. Soc. 2002. 23. 749. (b) Kim. S.-M. Jun. T.G. Sinth. Commun. 2002. 32. 3851 .

2. Meyers. A. I. Acc. Chem. Res. 1978. H. 375.

3. Sugiyama. T.: Sugawara. H.: Watanabe. M.: Yamashita. K. Agric. Biol. Chem. 1984, 48. 1841.

4. (a) For a review. see Lohray. B. B. Symhesis 1992,1035 . (b) Cho. G. Y.: Choi. J. Y.: Ko. S. Y. Bull Kowam Chem. Soc. 2002. 23. 11

5. Lohray. B. B.: Sharpless. K. B. Tetrohe dron Lett. 1989.30.2623.

6. (a) Homeyer. A. H U. S. Patent 2,399. 118. C. A. 1946. 40.4084. (b) Newmant. M. S.: Kutner. A. T. J. Am. Chem. Soc. 1951. 73. 4199. (c) Kang, S. H.: Hwang. Y. S.; Lee. H. S. Bull Kowan Chem. Soc. 2002, 23. 1195.

7. Evans, D. A.: Ennis. M. D; Mathre, D. J. J. Am. Chem Soc. 1982. 10+. 1737

8. Crimnins. M. T.: King. B. W. Tabet. E. A. J. Ant. Chent. Soc 1997. 119.7883.

9. Evans, D. A.: Britton. T. C.: Ellman, J. A. Tetrahedron Lett. 1987. 28. 6141 .

10. Gage. J. R.: Evans, D. A. Org. Sm. 1990. 68. 83

11. Evans. D. A.: Britton. T. C.: Ellman. T. A. Tetrahedron Lett. 1987. 28.6141.

12. Evans. D. A.: Nelson1. J. V: Vogel. E.: Taber. T. R. J. Ant Chem Soc. $1981,103,3099$. 